\title{
Structural monitoring for lifetime extension of offshore wind monopiles: Validation of load extrapolation algorithm
}

\author{
Lisa Ziegler $^{\text {a,b,* }}$, Nicolai Cosack ${ }^{a}$, Athanasios Kolios ${ }^{c}$, Michael Muskulus ${ }^{b}$ \\ ${ }^{a}$ Ramboll Wind, 20097 Hamburg, Germany \\ ${ }^{\mathrm{b}}$ Department of Civil and Environmental Engineering, Norwegian University of Science and \\ Technology NTNU, 7491 Trondheim, Norway \\ ${ }^{\mathrm{c}}$ Offshore Energy Engineering Centre, Cranfield University, Bedford MK43 0AL, UK
}

*Corresponding author: lisa.ziegler@ramboll.com

\begin{abstract}
Lifetime extension needs low-cost assessments that can identify the remaining useful life of offshore wind monopiles. A novel concept for load monitoring was developed that only needs strain gauges installed at one level of the support structure. Damage equivalent loads were calculated from the strain measurements and extrapolated to other locations of the structure using aero-hydro-elastic simulations and a k-nearest neighbor regression algorithm. In this paper, the load extrapolation algorithm was validated with measured data from two offshore wind turbines. The turbines had strain gauges installed at a distance of approximately $15 \mathrm{~m}$ and $25 \mathrm{~m}$. Results show that monthly damage equivalent loads can be predicted with errors smaller than $4 \%$ based on measurement data only. These results are very promising and should motivate further research in this direction.
\end{abstract}

Keywords: lifetime extension; offshore wind turbine; load monitoring; monopile; strain gauge; fatigue; $k$-nearest neighbor

\section{Introduction}

Offshore wind is still a young industry. The majority of assets have been operational for a few years only while the design lifetime of offshore wind turbines and their support structure has been typically 20-25 years in the past. Nowadays, the industry prepares to design new offshore wind farms for a longer lifetime in order to lower the cost of energy. For existing offshore wind farms, extension of the service life is appealing for operators to increase return on investments. Lifetime extension is technically possible if the turbine as well as its support structure have sufficient structural reserves left. Monopiles form the majority of installed support structures today [1] and are subject of this study.

In general, monopiles may have structural reserves at the end of their design life if either loadings are lower or material resistances are higher than designed for. This can be, for 
example, due to conservatism in environmental parameters (e.g. soil, wind speeds), operational conditions (e.g. turbine downtime), or material properties (e.g. manufacturing tolerances). Technical assessments are necessary to prove that operating assets do not fall below required safety levels during lifetime extensions. According to DNV GL [2], these assessments can be analytical and practical, and/or data-driven. Low-cost solutions for lifetime extension assessments are desirable since it is uncertain beforehand whether a potential for lifetime extension can be confirmed or not.

Analytical assessments are renewed load simulations with updated design models of the wind turbine and support structure as presented by Ziegler and Muskulus [3]. Data gathered during the service life of the offshore wind turbine should be used to update design models as well as environmental and operational assumptions. Practical assessments are inspections on site, which is afflicted with significant costs and risks due to the offshore environment. In addition, Ziegler and Muskulus [4] showed that the probability of detecting decisive fatigue cracks in circumferential welds of monopiles is low. Data-driven assessments may include monitoring of loads or structural health. Load monitoring tracks the load history and enables a direct comparison between design loading and occurred loading to derive the remaining useful lifetime (RUL) of the structure.

Load monitoring of steel structures is established practice for aging infrastructure. As an example, full field strain measurements are applied to evaluate the remaining fatigue life of existing steel bridges by Zhou [5], Leander et al [6], and Frangopol et al [7]. Zhou [5] claims that strain measurements at existing bridges are more accurate for assessing RULs than analytical fatigue assessments, which typically result in overestimation of stress ranges. Leander et al [6] demonstrate how load monitoring with strain gauges can clarify the status in case analytical assessments and inspections yield different results. In the case of a Swedish railway bridge, analytical fatigue reassessments showed that the calculated fatigue life of the stringers was already exceeded while inspections with eddy-current and magnetic particle methods gave no detection of fatigue damage. A monitoring program with strain gauges confirmed the stress ranges calculated in the analytical assessment [6]. Frangopol et al [7] highlight the impact of possible sensor errors associated with electrical strain gauges on fatigue reliability assessments of a steel bridge. Current studies in the field focus on probabilistic fatigue life prediction using strain monitoring data [8] and methods to extrapolate results to structural areas where no sensors are installed $[9,10]$. In practice, it is not possible to monitor all areas of interest due to cost and access restrictions.

This is particularly relevant for monopiles of offshore wind turbines, for which large parts of the structure are under water and below mudline. It is possible to monitor these areas directly if the monopile is equipped with strain gauges before pile driving, which has been done in several projects for research and development purposes. Many of these projects, however, experience troubles with the survival rate of the strain gauges during pile driving. For existing assets, it is expensive (below water) or impossible (below mudline) to retrofit strain gauges. Therefore, it would be very advantageous to extrapolate measurements from a limited number of sensors to the entire structure. In the offshore wind industry, several researchers have investigated load monitoring strategies with a limited number of sensors using physical models or artificial intelligence. Model-based time-domain approaches include Kalman filters, joint input-state estimation, and modal expansion algorithms [11-13]. These methods aim to track the time history of the vibrations of the whole structure. Artificial intelligence algorithms typically work with 10-minute statistics, such as damage equivalent loads $[14,15]$. 
In many existing offshore wind farms, some assets have strain gauges already installed at one height of the structure, typically at the transition piece above water. This data is ready-touse for lifetime extension assessments at no additional costs. However, these measurements need to be extrapolated to the entire support structure. In our previous work, we have proposed a novel method for extrapolation of measured loads using a simulation model and statistical algorithm [16]. The objective of this paper is to validate the load extrapolation algorithm with measurement data. Two month of strain measurements from two offshore wind monopiles are used for this purpose.

The remainder of the paper is structured as follows: Section 2 describes the load extrapolation algorithm and the measurement data used in the validation study. Results of the validation are discussed in Section 3 and concluded in Section 4.

\section{Methodology}

\subsection{Fatigue loading of offshore wind turbines}

Offshore wind turbines operate in a complex environment with wind, waves, current, and various operational conditions. Aero- and hydrodynamic excitation causes long-term cyclic loading at the support structure. The loading history at a specific structural hot spot consists of load ranges with variable amplitudes, each occurring for a specific number of cycles. Cyclic loading restricts the fatigue life of the support structure. The fatigue limit state is often driving the design of monopiles [17].

It is common industry practice to perform fatigue analysis using SN-curves $[18,19,20]$. SN-curves specify how many load cycles of a specific amplitude a material can endure before failure. The Palmgren-Miner rule of linear damage accumulation is commonly applied to calculate fatigue damage [21]. This hypothesis allows simplifying a variable-amplitude load time series into a single damage equivalent load (DEL). DEL is a load range with constant amplitude that - when applied for a specific number of reference cycles - causes the same amount of fatigue damage as the original variable-amplitude load time series [15].

\subsection{Load extrapolation algorithm}

The developed load monitoring concept extrapolates DELs measured at one location to the entire structure. DELs between different locations of a structure are correlated through the vibrational modes of the structure. If the structure vibrates in one mode only, DELs at different location of the structure will be highly correlated.

Figure 1 (a) shows power spectral densities of strain measurements at the transition piece of an offshore wind turbine. Excitation frequencies are quasi-static contributions from wind forces, wave excitation, first fore-aft and side-side bending frequencies, rotational bladepassing frequencies (1P and 3P), and the second fore-aft and side-side bending frequencies. Consequently, for each DEL measured at one location, a range of DELs at another location can occur depending on how the structure vibrates. Ziegler et al [16] showed that this range of DELs has a well-defined lower bound with limited scatter for a monopile support structure. This allows the application of a statistical model to predict DELs at location B from measurements at location A. 
In our previous work, we suggested to combine aero-hydro-elastic simulations with an updated finite element model and a k-nearest neighbor regression algorithm for extrapolation of DELs [16]. The methodology is briefly outlined in the following. It consists of simulations, measurements, and extrapolation of measurements. The reader is referred to [16] for further details.

1. Simulations: Aero-hydro-elastic simulations are performed with a finite element model that represents the global dynamic behavior of the installed offshore wind turbine. The finite element model used in design should be updated with on-site measurements to ensure that modal properties (such as natural frequencies, mode shapes, and damping) are represented correctly.

Realistic environmental and operational conditions as input for the load simulations are assembled into load cases. Sources for information on realistic conditions are on-site measurements (e.g. wave buoy, met mast - if available), recordings from the turbine control and performance monitoring system (SCADA), and site assessments during design. For each load case, aero-hydro-elastic simulations in line with design requirements and current state-of-art should be performed [22].

10-minute time series of loads at specified locations are obtained from the simulations for each load case. The locations are the point at the structure, where sensors are installed (location A), and the desired location to extrapolate to (location B).

Rainflow counting is performed on each time series to obtain the load ranges $S_{i}$ and corresponding number of cycles $N_{i}$ [20]. Results are transformed into DELs with Equation $1 . N_{k}$ is the number of reference cycles, $m$ is the inverse slope of the considered $\mathrm{SN}$-curve, and $n$ is the number of load ranges.

$$
D E L=\left(\sum_{i=1}^{n} \frac{N_{i}}{N_{k}} S_{i}^{m}\right)^{\frac{1}{m}}
$$

2. Measurements: Bending strain is measured at one location of the structure through adequately installed sensors. An example of sensor types and placement is given in Section 2.3. The measured strains $\varepsilon$ are transformed into bending moments $M$ according to Equation 2. $E$ is the Young's modulus and $Z$ is the elastic section modulus. The time series are then split into 10-minute intervals. Rainflow counting is performed and DELs are obtained for each 10-minute time interval.

$$
M=E \cdot Z \cdot \varepsilon
$$

3. Extrapolation: The DELs obtained from aero-hydro-elastic simulations (step 1) are used to calibrate the k-nearest neighbor regression algorithm for load extrapolation. The k-nearest neighbor regression algorithm acts as transfer function between the measurement location and the locations of interest for extrapolation. DELs calculated for location $A$ (measurement location) are sorted ascending. This results in an array of 
DELs with the length equal to the number of load cases. Each DEL at location $A$ has a corresponding DEL at location $B$ (extrapolation location) obtained from the simulations for the considered load case. The sorting order from the DELs of location $A$ is applied to the DELs of location $B$ also.

The value of a DEL measured at location $A$ at the real structure is sorted into the ascending array of simulated DELs at this location. A number of neighbors of the simulated DELs at location $A$ are chosen including the corresponding DELs at location $B$. This choice purely depends on the absolute value of DEL; underlying environmental and operational are ignored. The DEL at the unmeasured location $B$ at the real structure is then predicted as the mean (or weighted mean) from the chosen, neighboring simulated DELs at this location.

The selection of neighbors can be altered if information on operational or environmental parameters is available, e.g. from the SCADA system. Only simulated neighbors with similar input conditions are then considered for the extrapolation.

\subsection{Measurement data}

In this paper, we analyzed measurement data from two offshore wind turbines on monopile foundations. Both are situated in the same wind farm and are typical variable-speed, pitch-controlled wind turbines. The location of the wind farm is a typical North Sea site with medium-range water depth. Two months of measurement data were available for both offshore wind turbines. The months are consecutive in the year and have similar operational conditions. Further information on the type of wind turbines and site are excluded for confidentiality purposes.

The wind turbines are equipped with the following sensor system:

- At the first turbine, electrical resistance strain gauges were installed at the transition piece of the support structure at two different heights. The distance between the two levels is approximately $15 \mathrm{~m}$. Figure 1 (c) shows the approximate position of the two sets of strain gauges at level 1 and level 2. On each level, four axial strain gauges are placed with $90^{\circ}$ spacing around the circumference of the transition piece. The setup is redundant which makes it possible to detect calibration errors and to identify the amount of noise in the measurements. Figure 1 (b) shows spacing and labeling of the strain gauges.

- The second turbine has electrical resistance strain gauges at the upper part of the transition piece (level 1) and upper part of the monopile below water (level 3). The distance between both levels is approximately $25 \mathrm{~m}$. The location of the strain gauges is illustrated in Figure 1 (c). On level 1, four axial strain gauges are placed similar to turbine 1. On level 3, only two axial strain gauges are placed at position C and D.

- The strain gauges were calibrated and compensated for temperature effects. The sampling resolution was $20 \mathrm{~Hz}$.

- $\quad$ 10-minute average values of power output, turbine status, yaw direction, and mean wind speed from the nacelle anemometer were obtained from the SCADA system. 


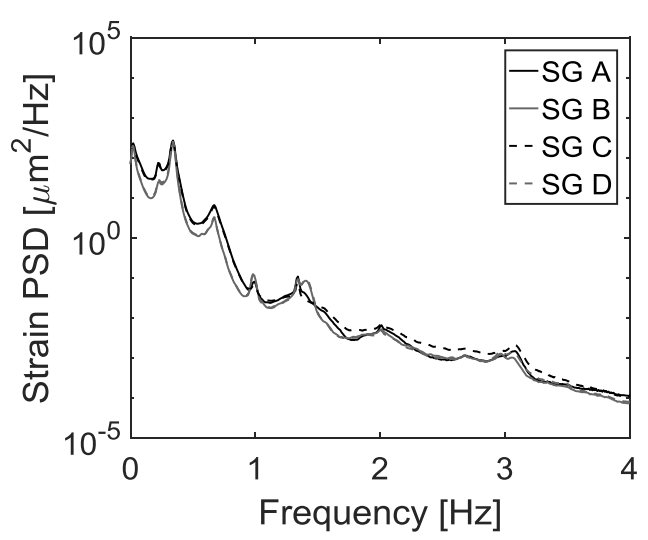

(a)

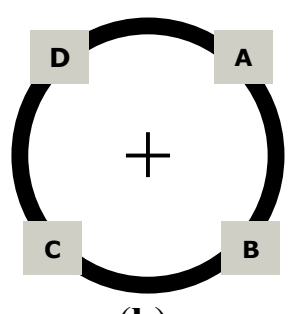

(b)

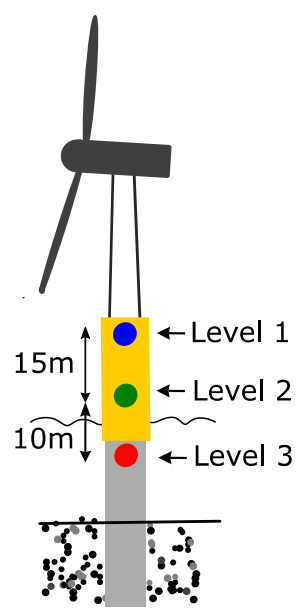

(c)

Figure 1. (a) Time series of strain measurements from four strain gauges at the transition piece of an offshore wind turbine are transformed into power spectral densities using Fast Fourier transform. (b) Four strain gauges are installed at level 1 and 2 . They are spaced in $90^{\circ}$ intervals. Level 3 only contains strain gauge C and D. (c) Schematic position of strain gauges at two offshore wind turbines used for the validation study. At one turbine, the strain gauges are installed at the transition piece at two levels. The distance between the two levels is approximately $15 \mathrm{~m}$. At a second turbine, strain gauges are installed at level 1 and at the upper part of the monopile (level 3). The distance between level 1 and level 3 is approximately $25 \mathrm{~m}$.

\subsection{Validation study}

The load extrapolation algorithm is validated with strain gauge data obtained from two offshore wind turbines as described in the previous sections. The validation is based on a data-only approach. This means that one month of the data was used to set up and calibrate the extrapolation algorithm. The performance of the algorithm was then tested with data from the second month. Consequently, the validation was decoupled from aero-hydro-elastic simulations in this work; only part (II) and (III) of the extrapolation algorithm were addressed (cf. Section 2.2).

Three validation studies were performed for turbine 1; two studies for turbine 2 . The validation studies are shown in Table 1. In case 1, the complete data set of DELs was used for the extrapolation. This includes all time periods for which strain measurements were uninterrupted during 10-minute intervals and time-synchronized SCADA data was available. In case 2, the extrapolation was performed conditional on power production recorded by the SCADA system. The algorithm was trained to distinguish between DELs recorded in three operational states: idling (mean produced power $\leq 0 \mathrm{~kW}$ ), rated power (mean produced power $\geq$ rated power), and the remaining conditions. For case 3 , the data set was cleaned by utilizing the redundancy of the sensor layout of turbine 1. Time series from strain gauges at opposite sides of the transition piece (A-C and B-D) should mirror each other. Therefore, the same DEL should be obtained from both sensors under ideal conditions. The difference between DELs from opposing strain gauges was evaluated to obtain an estimate of the noise level of 
the measurements. In the cleaned data set, only $95 \%$ of the DELs were further processed in the validation study. 5\% of the DELs with the largest differences between opposing strain gauges were deleted from the data set.

Table 1. Validation studies of the load extrapolation algorithm performed for turbine 1 and turbine 2.

\begin{tabular}{cccc}
\hline Case & Description & Turbine 1 & Turbine 2 \\
\hline 1 & Extrapolation of DELs & $\mathrm{X}$ & $\mathrm{X}$ \\
2 & Extrapolation of DELs with filter for power production & $\mathrm{X}$ & $\mathrm{X}$ \\
3 & Extrapolation of DELs with cleaned data set & $\mathrm{X}$ & \\
\hline
\end{tabular}

\section{Results and discussion}

\subsection{Processing of measurement data}

The measured data was checked for measurement noise and plausibility. Figure 2 (a) presents the time series of raw strain data of turbine 1 from the strain gauges B and D during one day. The time series show an opposing behavior. Figure 2 (b) shows the DELs obtained from the time series in Figure 2 (a) after rainflow counting. The DELs show a good match with a mean absolute percentage error of $3.0 \%$ for this day. This indicates that the sensors perform well with little measurement noise. The DELs measured by strain gauge D are slightly higher than from strain gauge B indicating small gain differences in the calibration of both strain gauges.

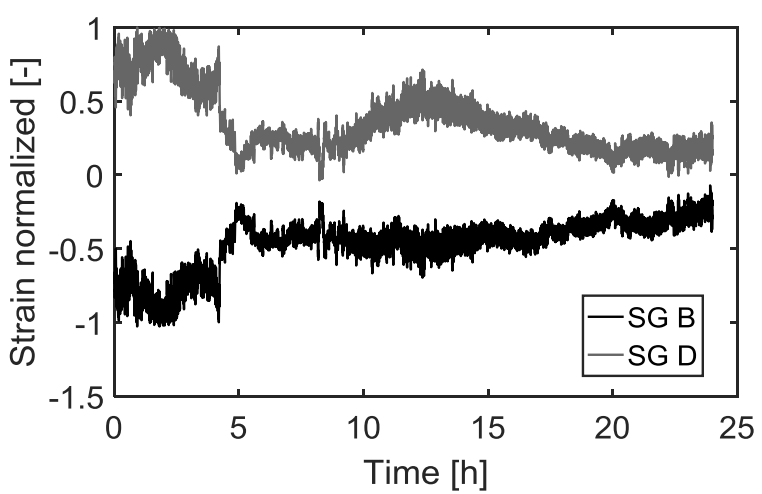

(a)

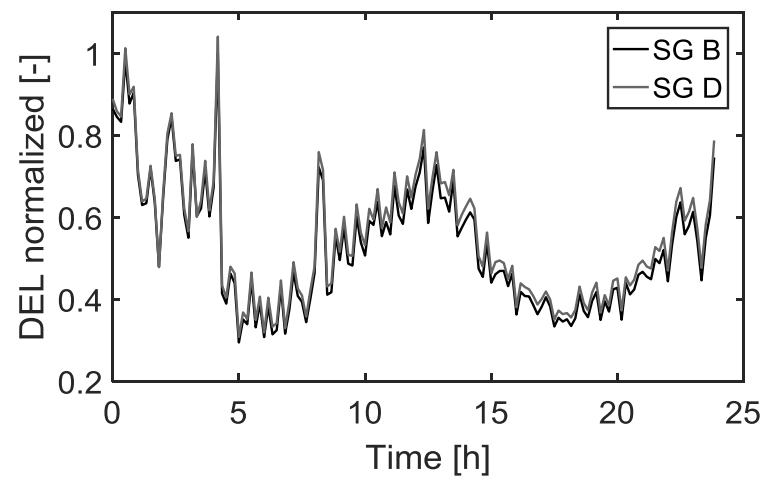

(b)

Figure 2. Strain gauge (SG) data from turbine 1. The data is normalized to the maximum of the time series. (a) 24 hours of measurements from strain gauges at opposite positions of the circumferential of the transition piece. (b) 10-minute DELs from opposing strain gauges after rainflow counting. The difference between the DELs from the two strain gauges is small.

Figure 3 (a) shows the difference between strain gauges $\mathrm{B}$ and $\mathrm{D}$ for the two measurement locations at the transition piece for one month. The differences between the strain gauge at the upper location are higher than for the lower location. On average, the differences are below zero at both levels which indicates that there is a small gain error in the calibration in line with Figure 2 (b). 
In Figure 3 (b) the measured mean fore-aft bending moment from 10-minute time intervals is plotted as a function of mean wind speed from the SCADA system. The data was selected so that the turbine rotor is facing in approximately the same direction as the strain gauge. For this example, the strain gauge is located at $315^{\circ}$. All data points, where the yaw direction of the turbine lies between $305^{\circ}$ and $325^{\circ}$, are included in the plot. The mean bending moments at the height of the strain gauge resulting from a theoretical thrust curve are plotted as black line in the same figure. The theoretical thrust curve was estimated from a typical thrust coefficients for a turbine of that size and from basic geometry since no detailed information on thrust was available. The measurements follow the shape of the calculated bending moment due to thrust, thus it is concluded that the data is plausible to use for the validation study. Bending moments at wind speeds below $4 \mathrm{~m} / \mathrm{s}$ are higher than the theoretical value possibly due to turbulence, inaccuracy of wind speed measurements from the nacelle anemometer, or potential offsets in the calibration of the strain gauge.

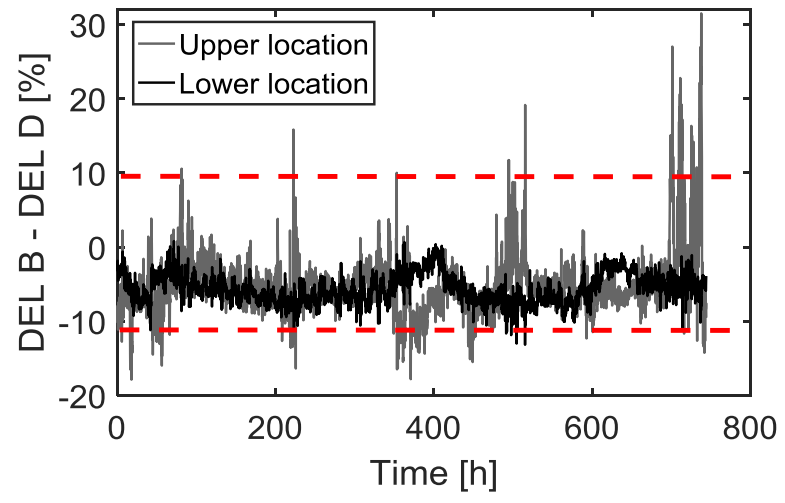

(a)

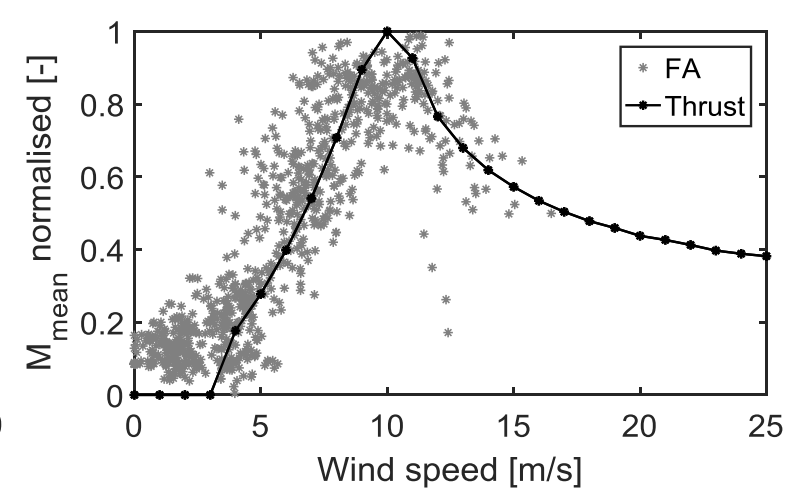

(b)

Figure 3. (a) Difference of DELs obtained from opposing strain gauges B and D of turbine 1 during one month in percent. Only 95\% of the DELs with the lowest differences between opposing strain gauges were considered in the cleaned data set. This corresponds to a threshold value of $\pm 10.3 \%$ (red line). (b) Mean fore aft (FA) bending moment (grey dots) from 10-minute time intervals as a function of wind speed. The calculated bending moments from a theoretical thrust curve of the turbine (black line) were estimated from turbine size and basic geometries. All data was normalized to the maximum of the theoretical thrust curve.

Table 2 shows the size of the data sets used in the validation study. Ideally, a month with $30 / 31$ days should have $4320 / 4464$ DELs recorded. The uncleaned data set of both turbines is smaller due to interruptions of strain measurements or missing SCADA data. The data availability of the uncleaned data set is above $94 \%$ for both turbines. The maximum differences between DELs from opposing strain gauges in the uncleaned data set were $39.0 \%$ and $855.9 \%$. The large difference of $855.9 \%$ belongs to a DEL that is very small $(<0.05$ $\mathrm{MPa}$ ), therefore the impact of sensor noise is large. For case 3 of the validation study (cf. Table 1), $5 \%$ of the DELs which have the highest differences were excluded from the data set. This corresponds to a threshold value of $\pm 10.3 \%$ differences between DEL B and DEL D that is allowed in the data set for this month. The threshold value is marked as red line in Figure 3 (a). This final data set used in the study is shown in Table 2. Only the data set for turbine 1 was cleaned since turbine 2 did not have opposing strain gauges for comparison. 
Table 2. Sizes of data set used in validation study. The cleaned data set is shown in brackets.

\begin{tabular}{lcccc}
\hline & \multicolumn{2}{c}{ Turbine 1 } & \multicolumn{2}{c}{ Turbine 2 } \\
\cline { 2 - 5 } & Month 1 & Month 2 & Month 1 & Month 2 \\
\hline No. of DELs & $4453(4230)$ & $4214(4003)$ & 4226 & 4115 \\
Data availability & $99.8 \%(94.8 \%)$ & $97.6 \%(92.6 \%)$ & $94.7 \%$ & $95.3 \%$ \\
Max. deviation & $39.0 \%(10.3 \%)$ & $855.9 \%(11.5 \%)$ & -- & -- \\
\hline
\end{tabular}

\subsection{Calibration of extrapolation algorithm}

Figure 4 (a) shows two months of DELs from turbine 1 sorted ascending for the upper measurement location. The lower location is approximately $15 \mathrm{~m}$ below. In Figure 4 (b) DELs are colored that correspond to idling and rated power conditions. Idling DELs were selected by filtering SCADA data for entries where power output is zero (black dots). Mean wind speed of these 10-minute time intervals is below the cut in wind speed of the turbine. Rated power DELs were filtered from SCADA also (red dots). All remaining data points (grey dots) correspond to mean wind speeds between cut-in wind speed and rated wind speed of the turbine. Both, rated power and idling conditions show less scatter than the remaining data points in Figure 4 (b).

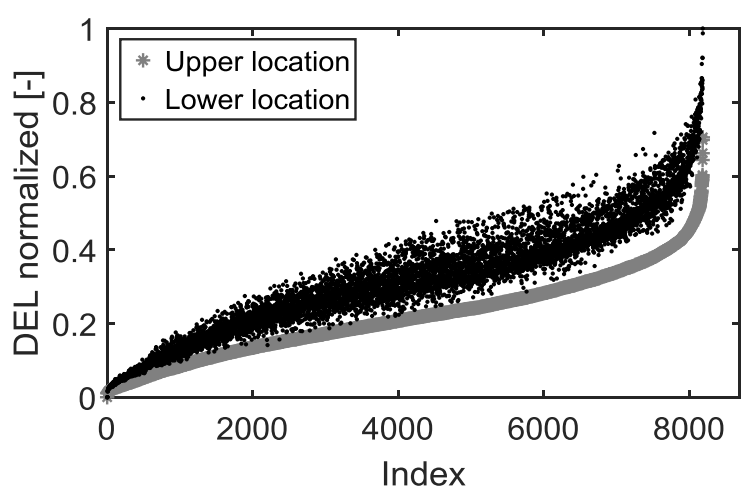

(a)

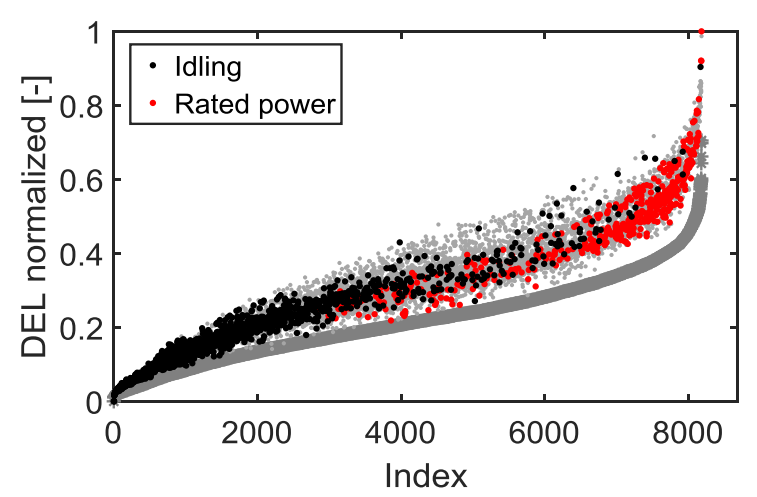

(b)

Figure 4. (a) Measured DELs at two locations of the structure of turbine 1 during two months. DELs are normalized to the maximum of the lower location. (b) DELs where the turbine 1 produces rated power are colored in red. The black colored DELs are idling conditions where the power output is zero and the average wind speed is below cut-in wind speed of the turbine. The remaining grey dots correspond to mean wind speeds between cut-in and rated wind speed.

\subsection{Extrapolation results}

Figure 5 (a) compares measured DELs and extrapolated DELs for turbine 1 (case 1) where the algorithm was calibrated with month $M 1$ and tested with month $M 2$ for one neighbor (red dots) and fifteen neighbors (light grey dots). The more neighbors are chosen, the more is the prediction smoothed in comparison to the scatter of the measurements. 
In Figure 5 (b) the histogram of the extrapolation results is shown for case 1. The algorithm was first calibrated with month $M 1$ to predict month M2. Afterwards, it was calibrated with month $M 2$ to predict month $M 1$. The cumulative DEL ratio for predicting month $M 1$ is 1.04; for prediction of month $M 2$ it is 0.96 .

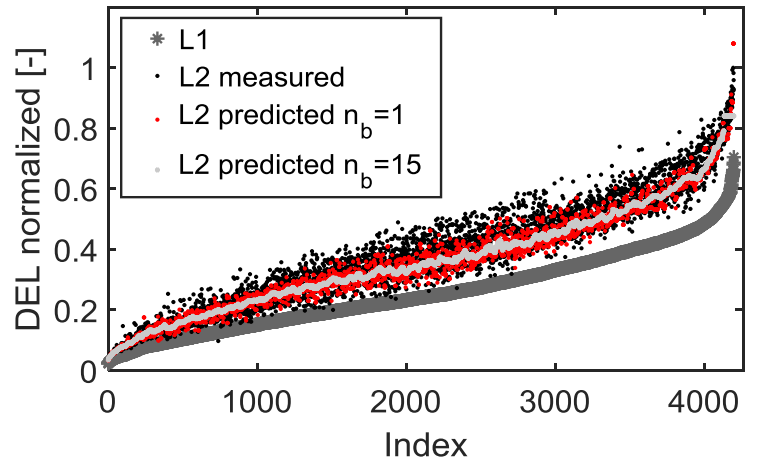

(a)

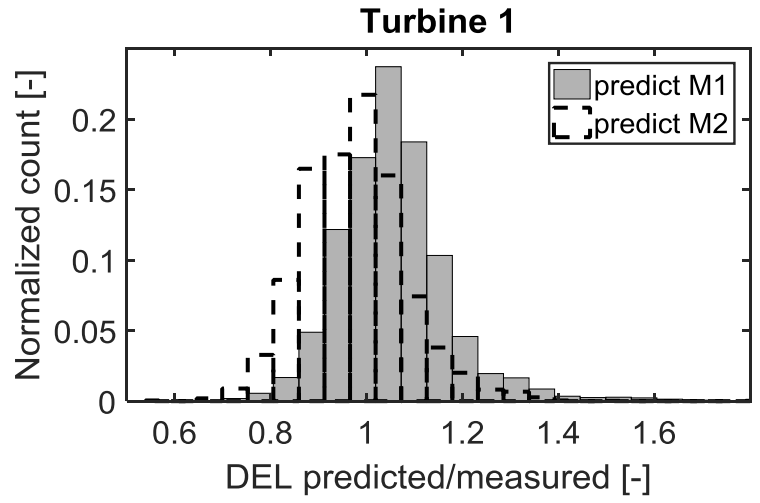

(b)

Figure 5. (a) Prediction of DELs from month $M 2$ with data from month $M 1$ for turbine 1 (case 1). $L 1$ is the upper and $L 2$ the lower location. DELs are normalized to the maximum of the lower location L2 measured. (b) Histogram of extrapolation results for calibration with month $M 1$ to predict month $M 2$ and vice versa for turbine 1 (case 1).

Figure 6 shows the monthly prediction errors in terms of cumulative DELs and damage for turbine 1 (a) and turbine 2 (b) for each case. The prediction error of damage is larger since it is exponentiated with the material parameter $m$ of the corresponding fatigue stress cycle curve (SN-curve). For turbine 1, the changes in prediction errors with the cleaned data set (case 3) were minor compared to the full data set (case 1). In addition, filtering for power production (case 2) yields comparable results to the unconditional extrapolation. This is possibly due to a counteracting effect: The filter reduces the scatter of DELs at the lower location, which is expected to improve the prediction. On the other hand, the prediction accuracy decrease with less data points available. The filter reduces the amount of data points in each category, which might counteract a potential improvement of the prediction due to the reduction of scatter. For turbine 2, the DEL prediction error of month M1 improves from $2.7 \%$ to $-2.0 \%$ while it does increase from $-0.4 \%$ to $0.9 \%$ for month $\mathrm{M} 2$ when filtering is applied. In line with turbine 1 , the effect of filtering was small in this case study. The influence of filters may be better once larger data sets are utilized.

Interestingly, the extrapolation results were better for turbine 2 than for turbine 1 , although the strain gauges are located at larger distance. This was unexpected since the extrapolation should be better the closer the sensors are (assuming that there are no local stress effects). It may indicate that the extrapolation is more sensitive to measurement noise than distance between locations. 


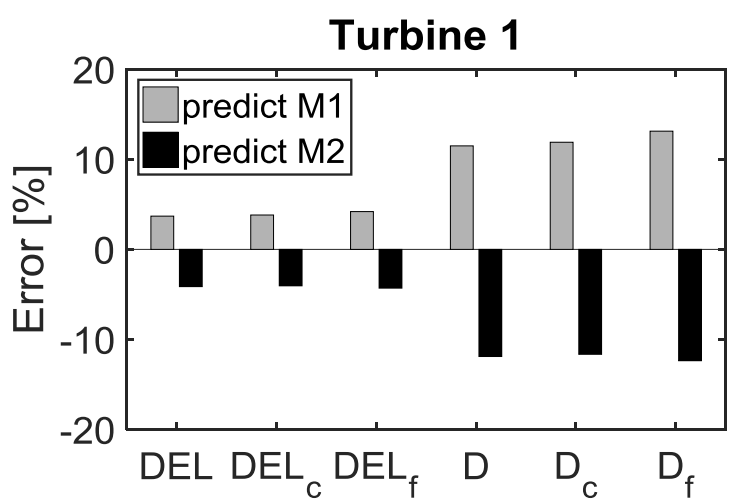

(a)

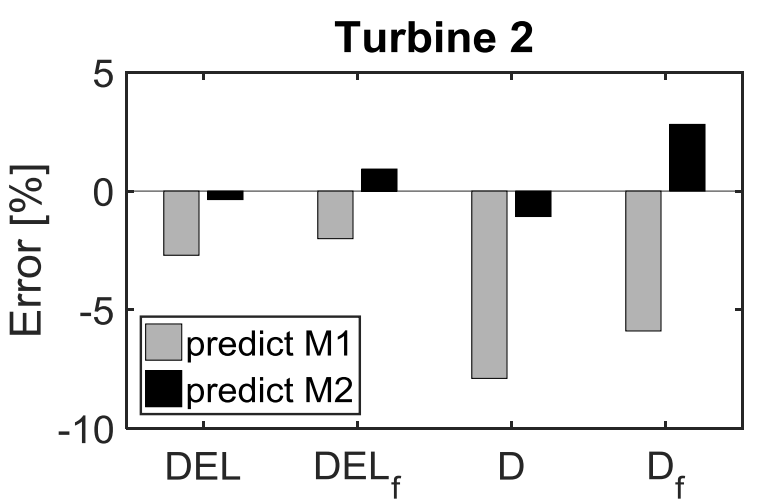

(b)

Figure 6. Prediction errors for monthly DELs and monthly damage (D) with and without filtering for produced power recorded by the SCADA system. Index $f$ denotes that a filter for produced power is applied (case 2). Index $c$ denotes that the data set was cleaned so that only $95 \%$ of recorded DELs were used (case 3). (a) Results for turbine 1 with strain gauges $15 \mathrm{~m}$ apart. (b) Results for turbine 2 with strain gauges $25 \mathrm{~m}$ apart.

\subsection{Discussion}

The validation study has successfully confirmed that the novel load extrapolation method is applicable to measurement data from two offshore wind monopiles. Prediction errors are in the same order of magnitude compared to results from tests with simulation data-only as performed previously by Ziegler et al [16]. Extrapolations with simulated data, where artificial measurement noise was imposed on the time series, resulted in an error of $4 \%$ for estimation of DELs [16]. This accuracy is very promising for fast and cheap evaluation of the loading history of a monopile and thus lifetime extension assessments.

Main limitations of the work are the accuracy of strain measurements. According to Martinez-Luengo et al [23], strain gauges are a mature technology with easy installation; however, the sensors are not very robust, have a short service life, and the installation is sensitive to misalignments. If strain data from the measured location has a large level of noise or includes measurement errors, the extrapolated DEL will also yield unrealistic results. A redundant setup of strain gauges (installed at opposite sides of the circumferential of the structure) helps to assess measurement noise and calibration offsets quickly. In the long-term, reliability of strain gauges might become an issue requiring extrapolation from some (representative) measurement years. In an application for lifetime extension, the load extrapolation algorithm would need to be calibrated with aero-hydro-elastic simulations instead of measurement data for locations without sensors. This might add additional prediction errors and has to be addressed in future work.

\section{Conclusion and future work}

Lifetime extension requires low-cost approaches to assess the remaining useful lifetime of offshore wind monopiles. For this purpose, a novel method to extrapolate loads from one level of strain gauges to the entire monopile is proposed by the authors and validated with 
measurement data in this paper. We conclude that the simple and cheap monitoring concept is able to predict damage equivalent loads at unmeasured locations with good accuracy. In addition, results showed that the load extrapolation algorithm is insensitive to the distance between measured location and predicted location. The validation was performed with measurement data only here. In a next step, the calibration of the extrapolation algorithm with aero-hydro-elastic simulations from an updated finite element model should be validated with measurement data. In addition, further work is desirable to improve filtering of measurement data for noise and unphysical recordings if no redundant sensors are available.

\section{Acknowledgement}

This project received funding from European Union's Horizon 2020 research and innovation program under the Marie Sklodowska-Curie grant agreement No 642108. We thank the operator for providing measurement data for the study.

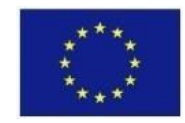

\section{References}

1. Ho, A.; Mbistrova, A. The European Offshore Wind Industry - Key Trends and Statistics 2016, Report, Wind Europe, Brussels, 2017.

2. DNV GL. Lifetime extension of wind turbines. DNVGL-ST-0262. 2016.

3. Ziegler, L.; Muskulus, M. Fatigue reassessment for lifetime extension of offshore wind monopile substructures. Journal of Physics: Conference Series 2016, 753(9); IOP Publishing, https://doi.org/10.1088/1742-6596/753/9/092010.

4. Ziegler, L.; Muskulus, M. Lifetime extension of offshore wind monopiles: Assessment process and relevance of fatigue crack inspection. Proceedings of the 12th EAWE PhD Seminar, DTU Lyngby, Denmark; 2016.

5. Zhou, Y. Assessment of bridge remaining fatigue life through field strain measurement. $J$ Bridge Eng 2006, 11(6), https://doi.org/10.1061/(ASCE)1084-0702(2006)11:6(737)

6. Leander, J.; Andersson, A.; Karoumi, R. Monitoring and enhanced fatigue evaluation of a steel railway bridge. Engineering Structures 2010, 32(3), 854-863, https://doi.org/10.1016/j.engstruct.2009.12.011.

7. Frangopol, D.M.; Strauss, A.; Kim, S. Bridge reliability assessment based on monitoring. Journal of Bridge Engineering 2008, 13(3), 258-270, https://doi.org/10.1061/(ASCE)1084-0702(2008)13:3(258).

8. Farreras-Alcover, I.; Chryssanthopoulos, M.K.; Andersen, J.E. Data-based models for fatigue reliability of orthotropic steel bridge decks based on temperature, traffic and strain monitoring. International Journal of Fatigue 2017, 95, 104-119, https://doi.org/10.1016/j.ijfatigue.2016.09.019. 
9. Pasquier, R; D’Angelo, L; Goulet, JA; Acevedo, C; Nussbaumer A; Smith IF. Measurement, data interpretation, and uncertainty propagation for fatigue assessments of structures. Journal of Bridge Engineering 2016, 21(5), 04015087, https://doi.org/10.1061/(ASCE)BE.1943-5592.0000861.

10. Hajializadeh, D; Obrien, EJ; O’Connor, AJ. Virtual structural health monitoring and remaining life prediction of steel bridges. Canadian Journal of Civil Engineering 2017, 44(4), 264-273, https://doi.org/10.1139/cjce-2016-0286.

11. Maes, K; Iliopoulos, A; Weijtjens, W; Devriendt, C; Lombaert, G. Dynamic strain estimation for fatigue assessment of an offshore monopile wind turbine using filtering and modal expansion algorithms. Mechanical Systems and Signal Processing 2016, 76, 592-611, https://doi.org/10.1016/j.ymssp.2016.01.004.

12. Iliopoulos, A; Shirzadeh, R; Weijtjens, W; Guillaume, P; Van Hemelrijck, D; Devriendt, C. A modal decomposition and expansion approach for prediction of dynamic responses on a monopile offshore wind turbine using a limited number of vibration sensors. Mechanical Systems and Signal Processing 2016, 68, 84-104, https://doi.org/10.1016/j.ymssp.2015.07.016.

13. Fallais, D.J.M; Voormeeren, S.; Lourens, E. Vibration-based Identification of Hydrodynamic Loads and System Parameters for Offshore Wind Turbine Support Structures. Energy Procedia 2016, 94, 191-198, https://doi.org/ 10.1016/j.egypro.2016.09.222.

14. Smolka, U.; Cheng, P.W. On the design of measurement campaigns for fatigue life monitoring of offshore wind turbines, Proceedings of The Twenty-third International Offshore and Polar Engineering Conference, International Society of Offshore and Polar Engineers, 2013.

15. Cosack, N. Fatigue load monitoring with standard wind turbine signals, $\mathrm{PhD}$ thesis, Universität Stuttgart, Germany, 2011.

16. Ziegler, L.; Smolka, U.; Cosack, N; Muskulus, M. Brief communication: Structural monitoring for lifetime extension of offshore wind monopiles: can strain measurements at one level tell us everything?, Wind Energ. Sci. 2017, 2, 469-476, https://doi.org/10.5194/wes-2-469-2017.

17. Vorpahl, F.; Schwarze, H.; Fischer, T.; Seidel, M.; Jonkman, J. Offshore wind turbine environment, loads, simulation, and design. Wiley Interdisciplinary Reviews: Energy and Environment 2013, 2(5), 548-570.

18. Ziegler, L.; Muskulus, M. Comparing a Fracture Mechanics Model to the SN-Curve Approach for Jacket-Supported Offshore Wind Turbines: Challenges and Opportunities for Lifetime Prediction. ASME. International Conference on Offshore Mechanics and Arctic Engineering, Volume 6: Ocean Space Utilization; Ocean Renewable Energy:V006T09A054. https://doi.org/10.1115/OMAE2016-54915. 
19. DNV GL. Support structures for wind turbines. DNVGL-ST-0126. 2016.

20. DNV GL. Fatigue design of offshore steel structures. DNVGL-RP-C203. 2016.

21. Petersen, C. Stahlbau. Springer Fachmedien Wiesbaden, Wiesbaden. 2013.

22. IEC. Wind turbines - part 3: Design requirements for offshore wind turbines. International standard IEC 61400-3. Geneva: International Electrotechnical Commission. 2009.

23. Martinez-Luengo, M.; Kolios, A.; Wang, L. Structural health monitoring of offshore wind turbines: A review through the Statistical Pattern Recognition Paradigm, Renewable and Sustainable Energy Reviews 2016, 64, 91-105, https://doi.org/10.1016/j.rser.2016.05.085. 